\title{
Pseudo-dipole signal removal from WMAP data
}

\author{
LIU Hao ${ }^{1 *} \&$ LI TiPei ${ }^{1,2,3 *}$ \\ ${ }^{1}$ Key Laboratory of Particle Astrophysics, Institute of High Energy Physics, Chinese Academy of Sciences, Beijing 100049, China; \\ ${ }^{2}$ Department of Physics and Center for Astrophysics, Tsinghua University, Beijing 100084, China; \\ ${ }^{3}$ Department of Engineering Physics and Center for Astrophysics, Tsinghua University, Beijing 100084, China
}

Received September 25, 2010; accepted October 11, 2010

\begin{abstract}
We have discovered in our previous work that different observational systematics, e.g. errors from antenna pointing directions or an asynchronism between the attitude and science data may generate a pseudo-dipole signal in the full-sky maps of the cosmic microwave background (CMB) anisotropy that have been published by the Wilkinson Microwave Anisotropy Probe (WMAP) team. We have found that the antenna sidelobe response to the Doppler signal produces a similar effect. In this work, independent from the sources, we have uniformly modeled the pseudo-dipole signal and have removed it from the published WMAP7 CMB maps by model fitting. The result demonstrates that the released WMAP CMB quadrupole is almost completely artificial.
\end{abstract}

cosmology, cosmic microwave background, data analysis

Citation: $\quad$ Liu H, Li T P. Pseudo-dipole signal removal from WMAP data. Chinese Sci Bull, 2011, 56: 29-33, doi: 10.1007/s11434-010-4222-0

\section{Pseudo-dipole signal}

The Wilkinson Microwave Anisotropy Probe (WMAP) mission makes measurements of temperature with two antennas, $\mathrm{A}$ and $\mathrm{B}$, and records, in time-order, the difference between the two antenna temperatures, $T_{\mathrm{A}}-T_{\mathrm{B}}$, which is called the time-order data (TOD). The observed cosmic microwave background (CMB) signal is contaminated by the Doppler effect, which is induced by the joint motion of the solar system and the spacecraft. The aroused dipole signal difference can be calculated by

$$
d=\frac{T_{0}}{c} \boldsymbol{v} \cdot\left(\boldsymbol{n}_{\mathrm{A}}-\boldsymbol{n}_{\mathrm{B}}\right),
$$

where $T_{0}=2.725 \mathrm{~K}$ is the $\mathrm{CMB}$ monopole, $c$ is the speed of light, $\boldsymbol{v}$ is the joint velocity, $\boldsymbol{n}_{\mathrm{A}}$ and $\boldsymbol{n}_{\mathrm{B}}$ are the unit direction vectors of the antenna $A$ and $B$, respectively [1]. In this paper, the vectors $\boldsymbol{v}, \boldsymbol{n}_{\mathrm{A}}$ and $\boldsymbol{n}_{\mathrm{B}}$ are defined in the spacecraft coordinate system. The errors in the line-of-sight (LOS) vectors, $\Delta \boldsymbol{n}_{\mathrm{A}}$ and $\Delta \boldsymbol{n}_{\mathrm{B}}$, produce a pseudo-dipole difference

*Corresponding authors (email: liuhao@ihep.ac.cn; litp@tsinghua.edu.cn) signal:

$$
d^{*}=\frac{T_{0}}{c} \boldsymbol{v} \cdot\left(\Delta \boldsymbol{n}_{\mathrm{A}}-\Delta \boldsymbol{n}_{\mathrm{B}}\right)=\frac{T_{0}}{c} \boldsymbol{v} \cdot \Delta \boldsymbol{n} .
$$

The dipoles for each of the observations must be removed from the raw data before map-making because their intensities are roughly 10 to 20 times greater than those of the CMB anisotropies. A small error in antenna direction produces an error in predicted dipole intensity, which then causes a noticeable pseudo-dipole signal in the resulting CMB map through the Doppler dipole subtraction process. A pure time-ordered dipole signal produces only a dipole component in the final CMB map, whereas a time-ordered pseudo-dipole signal may produce other components as well, in particular the quadrupole, on the final CMB map, as shown in Figure 1. For example, a LOS error of $\sim 7^{\prime}$, corresponding to approximately a half-pixel in the WMAP resolution, can cause the dipole signal to deviate by as much as 10-20 $\mu \mathrm{K}$, which cannot be ignored when compared to the relatively weak CMB signal. An asynchronism between the attitude and differential data can also produce the pseudo-dipole signal. The WMAP mission uses two separate 
clocks, one for the attitude data and the other for the science data. We have found that there exists an undocumented timing offset between the spacecraft attitude and radio flux density timestamps in the Meta and science data tables. The attitude datum is recorded $25.6 \mathrm{~ms}$ later than the corresponding differential datum [2], which can produce the same effect as a constant LOS error in spacecraft coordinates. Roukema has shown that the $25.6 \mathrm{~ms}$ timing offset actually exists in the calibration process of the TOD data by the WMAP team. This incorrectly calibrated TOD may cause a significant overestimation of the CMB quadrupole amplitude [3].

\section{Sidelobe pickup}

Another possible source of pseudo-dipole signal in the released WMAP maps is the sidelobe signal contamination. Like all radio telescopes, the WMAP antennas have both main beam response and sidelobe response. The WMAP antenna sidelobe response has been described in [4] and the corresponding data file is publicly available ${ }^{1)}$. The data file contains full sky maps in spacecraft coordinates, in which the sidelobe responses are given in normalized gain $G$, where the normalization rule is that the summation of all gains for one antenna (including the main beam) equals $N$, the number of pixels in the map. Thus, for each differential observation, the recorded difference signal is $\sum_{i=0}^{N-1}\left(G_{i}^{\mathrm{A}}-G_{i}^{\mathrm{B}}\right) T_{i} / N$. Let $k$ denote the pixels corresponding to a specific sidelobe and then the overall sidelobe response is $\sum_{k}\left(G_{k}^{\mathrm{A}}-G_{k}^{\mathrm{B}}\right) T_{k} / N$. The overall sidelobe response should contribute to a pseudo component of the differential Doppler signal:

$$
d_{\text {sidelobe }}^{*}=\frac{T_{0}}{c} \boldsymbol{v} \cdot \sum_{k} \frac{\left(G_{k}^{\mathrm{A}}-G_{k}^{\mathrm{B}}\right) \boldsymbol{n}_{k}}{N} .
$$

The spacecraft LOS vectors, as well as $\boldsymbol{n}_{k}$, are constant vectors in spacecraft coordinates. Because $G$ is a normalized gain, it can also be treated as constant. Therefore, we have two constant vectors: $\Delta \boldsymbol{n}_{\mathrm{A}}^{*}=\sum_{k} G_{k}^{\mathrm{A}} \boldsymbol{n}_{k} / N$ and $\Delta \boldsymbol{n}_{\mathrm{B}}^{*}=$ $\sum_{k} G_{k}^{\mathrm{B}} \boldsymbol{n}_{k} / N$. Because the sidelobe gain is much smaller than the main lobe gain, $\Delta \boldsymbol{n}_{\mathrm{A}}^{*}$ and $\Delta \boldsymbol{n}_{\mathrm{B}}^{*}$, are both small, constant vectors, and

$$
d_{\text {sidelobe }}^{*}=\frac{T_{0}}{c} \boldsymbol{v} \cdot\left(\Delta \boldsymbol{n}_{\mathrm{A}}^{*}-\Delta \boldsymbol{n}_{\mathrm{B}}^{*}\right)=\frac{T_{0}}{c} \boldsymbol{v} \cdot \Delta \boldsymbol{n}^{*} .
$$

Comparing eqs. (2) and (4), we can see that the entire sidelobe Doppler pickup can be exactly described by a small constant deviation in the differential spacecraft LOS vector. Similarly, the effect of the sidelobe response uncer- tainty on the differential Doppler dipole is also equivalent to introducing a small differential LOS error, $\Delta \boldsymbol{n}^{*}$. It is important to note that, even if the LOS is absolutely accurate, this pseudo-dipole signal from the sidelobe response uncertainty can still exist.

The amplitude of the equivalent LOS error, induced by sidelobe Doppler pickup, can be estimated by arcsin $\left(\left|\Delta \boldsymbol{n}_{\mathrm{A}}^{*}\right| /\left|\Delta \boldsymbol{n}_{\mathrm{A}}\right|\right)$, which ranges between $\sim 50^{\prime}$ and $75^{\prime}$ for different bands. The WMAP team believes that the Galactic sidelobe pickup is negligible for all bands except the $\mathrm{K}$-band. The reason is that the Galactic emission is much weaker in other bands than in the K-band [4]. The situation is significantly different for the Doppler signals because they have almost the same amplitude in all bands. Moreover, the Doppler signal is as strong as several $\mathrm{mK}$ over the entire sky, whereas the Galactic emission is strong only for low Galactic latitudes and the strength decreases rapidly for higher latitudes. Thus, the overall power of the Doppler signal is about $10-40$ times higher than the overall Galactic foreground emission power. The effect of the sidelobe Doppler signal pickup is at least 10 times stronger than the sidelobe Galactic pickup.

The WMAP antenna sidelobe gain patterns are estimated by ground-based measurements and in-flight lunar measurements. For the K-band, the WMAP first-year in-flight gain measurements are $60 \%$ systematically brighter than ground-based measurements. The WMAP team scaled up the ground-based measurements by $30 \%$ and scaled down the lunar results by $30 \%$ to yield a best-guess sidelobe gain map. In Sections 2.4 and 4 of [4], the overall calibration uncertainty is estimated as $30 \%$, and thus the amplitude of the equivalent LOS error, induced by the sidelobe Doppler pickup uncertainty, could be as much as approximately $15^{\prime}-22^{\prime}$ (estimated by $\left.\arcsin \left(\left|\Delta \boldsymbol{n}_{\mathrm{A}}^{*}\right| /\left|\Delta \boldsymbol{n}_{\mathrm{A}}\right|\right) \times 30 \%\right)$, which is much more than enough to produce considerable pseudodipole contamination. From the WMAP releases, we find that the WMAP7 sidelobe file is exactly the same as WMAP1 for the K-band. For other bands, the sidelobe files of a single survey year are slightly changed from year-1 to year-7, indicating that the accuracies have not been effectively improved. We can see from the above analysis that the pseudo-dipole contamination from the sidelobe response uncertainty cannot be ignored and must be removed from the published WMAP maps.

\section{Modeling and removing pseudo-dipole signal}

The approach we used to remove the pseudo-dipole signal is same as the WMAP foreground removal. Different effects arising in the foreground are separately modeled with template maps: the $94 \mathrm{GHz}$ dust map $t_{\mathrm{d}}$ for dust emission [5], 
the full-sky $\mathrm{H}_{\alpha}$ map $t_{\mathrm{H}}$ for free-free emission [6], and the synchrotron template $t_{\mathrm{s}}$ derived from $\mathrm{K}$, Ka bands for synchrotron emission [7,8]. The foreground emission is removed with model fitting by the WMAP team $[7,8]$ to obtain the clean temperature:

$$
t_{\text {clean }}=t^{\prime}-\left(c_{\mathrm{d}} t_{\mathrm{d}}+c_{\mathrm{H}} t_{\mathrm{H}}+c_{\mathrm{s}} t_{\mathrm{s}}\right)
$$

where the coefficients $c_{\mathrm{d}}, c_{\mathrm{H}}$ and $c_{\mathrm{s}}$ are determined by weighted fitting to make $t_{\text {clean }}^{2}=\min$.

The pseudo-dipole signal in WMAP temperature maps can be removed with the same approach as for foreground contamination. The overall effect of the three systematics, mentioned in Sections 1 and 2, LOS error, timing offset, and sidelobe Doppler pickup, can be generally described by an overall deviation $\Delta \boldsymbol{n}$ from the true differential antenna direction. The deviation, which is induced by the pseudodipole signal in a released WMAP map, can be easily modeled. For each measured temperature difference in the TOD used to produce the map, we substitute the pseudo-dipole signal, calculated from eq. (2) for an assumed $\Delta \boldsymbol{n}$, to produce a new TOD. In the calculation, after adding a deviation to the LOS vector, we continually re-scale the LOS vector to keep it unitary. The temperature map produced by map-making ${ }^{2)}$ from the new TOD can be used as a model of the pseudo-dipole signal in the released map. In the calculation, we use the spacecraft coordinate system $(X, Y, Z)$, where the $X$-axis is parallel to plane of radiators, the $Z$-axis is the anti-sun direction of the spin axis, and the $Y$-axis is perpendicular to both. The WMAP spacecraft scans the sky with a hybrid motion mode consisting of rotation and precession. In spacecraft coordinates, the LOS unit vectors of its two antennas are close to $(x, y, z)=(0,0.94,-0.33)$ and $(x$, $y, z)=(0,-0.94,-0.33)$, the spacecraft rotation being around the $Z$-axis. Suppose the overall LOS error $\Delta \boldsymbol{n}$ from all such effects is made up of three small vectors $(\delta, 0,0),(0, \delta, 0)$ and $(0,0, \delta)$, with $\delta=0.01$. Each of these alone on the final map induces its own full-sky distribution of deviation $\delta t_{x}$, $\delta t_{y}$ and $\delta t_{z}$, respectively. The induced deviation from the released WMAP7 year-1 Q1-band map $\delta t_{x}, \delta t_{y}$ and $\delta t_{z}$ are shown in Figure 1. The results for other years and other bands are similar.

With the deviation templates $\delta t_{x}, \delta t_{y}$ and $\delta t_{z}$, the clean full sky temperature map should be

$$
t_{\text {clean }}=t^{\prime}-\left(c_{x} \delta t_{x}+c_{y} \delta t_{y}+c_{z} \delta t_{z}\right) .
$$

Generally speaking, we should use all three templates $\delta t_{x}$, $\delta t_{y}$ and $\delta t_{z}$ in fitting; however, the case for WMAP is slightly different. It is clear from Figure 1 that $\delta t_{y}$ and $\delta t_{z}$ are highly correlated, and thus we can use two templates, $\delta t_{x}$ and $\delta t_{y z}=\left(\delta t_{y}+\delta t_{z}\right) / 2$, instead of three to get a good enough result (it is possible to further improve the fitting by all three templates and fine tuning the coefficients based on the result of two-template fitting, but this does not make much difference). Using two templates in fitting, we can also avoid possible degeneracy issues, and thus the derived clean full sky temperature map for WMAP should be

$$
t_{\text {clean }}=t^{\prime}-\left(c_{x} \delta t_{x}+c_{y z} \delta t_{y z}\right)
$$

where $t^{\prime}$ is the corresponding WMAP CMB temperature map and the coefficients $c_{x}$ and $c_{y z}$ can be determined by minimizing $t_{\text {clean }}^{2}$. Using the standard IDL program "regress", we find that $c_{x}=-0.35, c_{y z}=-0.78$ for the WMAP7 year-1 Q1-band map. We also model and remove the pseudo-dipole signal from the released WMAP7 year-1 to year-7 maps of the Q1, Q2, V1, V2, W1, W2, W3 and W4 bands, separately ${ }^{3)}$. From the clean maps, we calculate the power spectra and residual quadrupoles. Table 1 lists the obtained residual quadrupoles for different bands, where the average residual quadrupole (line 2) and the sample standard deviation (line 3) are calculated from the 7 single year results for each band. The overall average clean quadrupole


Figure 1 Induced map from the pseudo-dipole signal produced by an overall direction error $\Delta \boldsymbol{n}$, for WMAP7 year-1 Q1-band, in mK and in Galactic coordinates. From left to right: $\delta t_{x}$ with $\Delta \boldsymbol{n}=(0.01,0,0), \delta t_{y}$ with $\Delta \boldsymbol{n}=(0,0.01,0)$, and $\delta t_{z}$ with $\Delta \boldsymbol{n}=(0,0,0.01)$. The results for other years and other bands are similar.

2) Our map-making codes are publicly released on the website of Tsinghua Center for Astrophysics at http://dpc.aire.org.cn/data/wmap/09072731/release_v1/source_code/v1/ and on the CosmoCoffee Forum at http://cosmocoffee.info/viewtopic.php?p=4525\#4525.

3) For example, if the two templates $\delta t_{x}$ and $\delta t_{y}$ are derived from WMAP7 year-1 Q1 band, then they are used to fit the WMAP7 year-1 Q1 band single year CMB temperature map. The WMAP7 single year CMB temperature maps can be found at http://lambda.gsfc.nasa.gov/product/map/dr4/maps_forered_da_r9_i_1yr_get.cfm. 
Table 1 The residual quadrupole power $\left(\mu \mathrm{K}^{2}\right)$

\begin{tabular}{ccc}
\hline Wave band & Residual quadrupole & Standard deviation \\
\hline Q1 & 16.0 & 7.0 \\
Q2 & 13.6 & 3.6 \\
V1 & 10.4 & 3.9 \\
V2 & 13.7 & 2.8 \\
W1 & 27.5 & 7.0 \\
W2 & 20.2 & 8.5 \\
W3 & 12.6 & 5.3 \\
W4 & 23.3 & 27.4 \\
\hline
\end{tabular}

power for all bands is found to be $\sim 17.1 \mu \mathrm{K}^{2}$, only $\sim 14 \%$ of what has been released by the WMAP team ${ }^{4}$, indicating that most of the published WMAP CMB quadrupole is artificial.

\section{Discussion}

We have found serious systematic errors in the released WMAP temperature maps $[9,10]$, which have been confirmed by other independent works (e.g. [11]). We have then developed a software package for WMAP data processing independently from the WMAP team and newly produced $\mathrm{CMB}$ maps, which are significantly different than the official maps [12]. Later, in searching for the source of the difference between our maps and the official maps, we have discovered a $-25.6 \mathrm{~ms}$ asynchronism between the spacecraft attitude and the radiometer output timestamps in the official WMAP Meta and science data tables. Such a timing-offset, if not properly corrected in data processing, could generate serious consequences in the recovered CMB map and power spectrum [2]. The antenna sidelobe response to the Doppler signal has been found to produce similar effect as well. We find that these systematic effects induce a pseudo-dipole signal in WMAP raw differential temperature data, which can be summarized in a uniform description, namely the overall differential direction error $\Delta \boldsymbol{n}$. There could be other sources with similar attributes as these, yet a simple $\Delta \boldsymbol{n}$ can describe them all equivalently. For example, the LOS is determined by observing Jupiter; however, for each observation, the relative direction of Jupiter provides no more than three conditions: $(x, y, z)$, but we need four conditions in order to definitely determine the attitude of the spacecraft: $(x, y, z)$ and the rotation. What is more, the LOS is determined, together with the antenna main beam response; therefore, the uncertainty of the beam response is also included in the LOS uncertainty.

Moreover, the WMAP team themselves have found that some systematic effects can produce quadrupole-like deviations in the final CMB map ([13], Figure 3). Recently,
Roukema [3,14] have pointed out that a small timing error during calibration of the raw TOD could correspond to the addition of a pseudo-dipole difference signal with no effect on positional data.

It is impossible to exactly estimate all such effects, especially when not all effects are necessarily known, yet it is straight forward to model the impact of $\Delta \boldsymbol{n}$ upon the final CMB map and remove it by model fitting. It is apparently much more feasible and effective to employ this model fitting than it is to estimate and remove all possible sources one by one. What is important for model fitting is the feature of a template map, not the absolute amplitude. For example, the WMAP foreground removal will be the same even if the three foreground templates $t_{\mathrm{d}}, t_{\mathrm{H}}$ and $t_{\mathrm{s}}$ are all doubled. In this work, the result of removing a pseudodipole signal is not dependent on the amplitude that is assumed for the direction error. A larger amplitude leads to a lower fitted coefficient in model fitting so that the final correction to the CMB map is the same. It is noteworthy that the characteristic feature of the template map of the pseudo-dipole signal, induced by a pointing or timing error, which has been obtained in our previous work [2] (shown in Figure 1 of this work) has already been confirmed by other independent works $[3,15]$.

Our result demonstrates that a pseudo-dipole field can produce deviations in the sky temperature map with a structure uniquely similar to the CMB quadrupole pattern that has been published by the WMAP team. The template map of the pseudo-dipole induced temperature deviation, shown in Figure 1, is generated from a differential dipole field which is completely determined by the spacecraft velocity and overall-equivalent error of differential direction without using any CMB signal. The pseudo-dipole signal in the differential datum for a WMAP observation distorts the temperatures for corresponding sky pixels and varies from observation to observation. Therefore, the pseudo-dipole induced temperature map is generated by the pseudo-dipole signal, combined with the WMAP scan pattern, which is highly relative to the ecliptic plane $[9,16]$. Because the WMAP CMB quadrupole component is highly aligned and near the ecliptic plane, this old puzzle in cosmology can now be naturally explained by the pseudo-dipole signal effect. To continue to insist that the published WMAP quadrupole is actually cosmological in origin now becomes more difficult: the primordial density fluctuations not only occasionally need to be aligned in the plane of the solar system, but also occasionally must be in phase with the scan strategy of the WMAP mission.

The data analysis made use of the WMAP data archive and the HEALPix software package (Gorski K M, Hivon E, Banday A J, et al. Astrophys J, 2005, 622: 759-771). This work was supported by the National Natural

4) The WMAP pseudo- $C_{l}$ power spectrum can be found in the WMAP1 release $\left(123 \mu \mathrm{K}^{2}\right.$ for quadrupole $)$ at http://lambda.gsfc.nasa.gov/data/map/powspec/wmap binned_tt_powspec_yr1_v1p1.txt. With the same CMB maps, our pseudo- $C_{l}$ estimator provides a consistent low- $l$ power spectrum to the WMAP1 release, including the quadrupole. Although the WMAP team never gave a pseudo- $C_{l}$ quadrupole in later releases, we have tested and made sure that this value is almost the same in all releases from WMAP1 to WMAP7. 
Science Foundation of China (11033003), National Basic Research Program of China (2009CB824800) and the CAS project (KJCX2-YW-T03).

1 Hinshaw G, Weiland J L, Hill R S, et al. Five-year Wilkinson Microware Anisotropy Probe observations: Data processing, sky maps, and basic results. Astrophys J Suppl Ser, 2009, 180: 225-245

2 Liu H, Xiong S L, Li T P. The origin of the WMAP quadrupole. arXiv: $1003.1073 \mathrm{v} 2$

3 Roukema B F. On the suspected timing-offset-induced calibration error in the WMAP time-ordered data. arXiv: 1007.5307

4 Barnes C, Hill R S, Hinshaw G, et al. First-year WMAP observations: Galactic signal contamination from sidelobe pickup. Astrophys J Suppl Ser, 2003, 148: 51-62

5 Finkbeiner D P, Davis M, Schlegel D J, et al. Extrapolation of galactic dust emission at 100 microns to cosmic microwave background radiation frequencies using FIRAS. Astrophys J, 1999, 524: 867-886

6 Finkbeiner D P. A full-sky $\mathrm{H} \alpha$ template for microwave foreground prediction. Astrophys J Suppl Ser, 2003, 146: 407-415

7 Bennett C L, Hill R S, Hinshaw G, et al. First-year Wilkinson Microwave Anisotropy Probe (WMAP) observations: Foreground emission. Astrophys J Suppl Ser, 2003, 148: 97-117

8 Gold B, Bennett C L, Hill R S, et al. Five-year Wilkinson Microwave
Anisotropy Probe (WMAP) observations: Galactic foreground emission. Astrophys J Suppl Ser, 2009, 180: 265-282

9 Li T P, Liu H, Song L M, et al. Observation number correlation in WMAP data. Mon Not Roy Astron Soc, 2009, 398: 47-52

10 Liu H, Li T P. Systematic distortion in cosmic microwave background maps. Sci China Ser G-Phys Mech Astron, 2009, 52: 804-808

11 Aurich R, Lustig S, Steiner F. Hot pixel contamination in the CMB correlation function? Class Quantum Grav, 2009, 27: 095009

12 Liu H, Li T P. Inconsistency between WMAP data and released map. Chinese Sci Bull, 2010, 55: 907-909

13 Jarosik N, Barnes C, Greason M R, et al. Three-year Wilkinson Microwave Anisotropy Probe (WMAP) observations: Beam profile, data processing, radiometer characterization, and systematic error limits. Astrophys J Suppl Ser, 2007, 170: 263-287

14 Roukema B F. On the suspected timing error in Wilkinson Microwave Anisotropy Probe (WMAP) map-making. Astron Astrophys, 2010, 518: A34

15 Moss A, Scott D, Sigurdson K. Induced CMB quadrupole from pointing offsets. arxiv: 1004.3995

16 Hinshaw G, Nolta M R, Bennett C L, et al. Three-year Wilkinson Microwave Anisotropy Probe (WMAP) observations: Temperature analysis. Astrophys J Suppl Ser, 2007, 170: 288-334

Open Access This article is distributed under the terms of the Creative Commons Attribution License which permits any use, distribution, and reproduction in any medium, provided the original author(s) and source are credited. 